Cite this: Phys. Chem. Chem. Phys., 2013, 15, 6219

Received 28th January 2013, Accepted 11th March 2013

DOI: $10.1039 / \mathrm{c} 3 \mathrm{cp} 50388 \mathrm{~g}$

www.rsc.org/pccp

\title{
Monte Carlo simulation and free energies of mixed oxide nanoparticles
}

\author{
John A. Purton, ${ }^{\star a}$ Stephen C. Parker ${ }^{b}$ and Neil L. Allan ${ }^{c}$
}

\begin{abstract}
A Monte Carlo Exchange technique is used to study the thermodynamic properties of $\mathrm{MgO}-\mathrm{MnO}$ nanoparticles ranging in size from 1728 to 21952 ions. The solubility of $\mathrm{Mg}^{2+}$ is much greater in $\mathrm{MnO}$ than the reverse, reflecting the difference in size between the two cations. The solubility, for a given temperature, diminishes with nanoparticle size. As the $\mathrm{Mn}$ concentration is progressively increased the $\mathrm{Mn}^{2+}$ ions occupy the corners, edges and then surface sites of the nanoparticle before entering subsurface layers. We do not observe any pronounced ordering of the cations within the body of the nanoparticles themselves. The enthalpies of forming ternary nanoparticles from particles of $\mathrm{MgO}$ and $\mathrm{MnO}$ of the same size vary with the size of the nanoparticle and become more positive for a given concentration as the particle size increases. Free energies of mixing of the two end-member nanoparticles have been determined using the semigrand ensemble. The consolute temperature (the temperature above which there is complete miscibility) increases non-linearly with the size of the nanoparticle by approximately $70 \%$ over the size range considered.
\end{abstract}

\section{Introduction}

Heterogeneous nanostructures have become increasingly important, since they often exhibit multifunctional capabilities with tuneable or enhanced properties. ${ }^{1}$ Indeed, the formation of core-shell structures represents one of the most effective experimental approaches for creating heterostructured nanodevices for many technological applications such as high temperature alloys, catalysts and solid oxide fuel cells. ${ }^{2}$ The fabrication and internal structure (i.e. the distribution of atoms/ions) of these functional nanoparticles is controlled by a variety of parameters such as temperature, chemical environment, rate of growth and diffusion. Ferrando et $a .^{3}$ have reviewed comprehensively both theoretical and experimental studies of nanoalloy clusters and nanoparticles of metallic elements. Much of the theoretical work has focused on the calculation of the potential energy surface to determine the most stable configuration. Size and surface segregation effects have been considered analytically ${ }^{4}$ and Monte Carlo methods have been employed to calculate thermodynamic properties of

\footnotetext{
${ }^{a}$ STFC, Daresbury Laboratory, Warrington, WA4 4AD, UK.

E-mail:john.purton@stfc.ac.uk; Fax: +44 (0)1925 603100; Tel: +44 (0)1925 603785

${ }^{b}$ School of Chemistry, University of Bath, Claverton Down, Bath, BA2 7AY, UK. E-mail: s.c.parker@bath.ac.uk; Fax: +44 (0)1225 386231; Tel: +44 (0)1225 386505

${ }^{c}$ School of Chemistry, University of Bristol, Cantock's Close, Bristol, BS8 1TS, UK.

E-mail: n.l.allan@bris.ac.uk; Fax: +44 (0)117 925 1295; Tel: +44 (0)117 9288308
}

metal nanoparticles, for example the excess enthalpy of mixing ${ }^{5}$ and segregation of atoms to form core-shell structures. ${ }^{5-10}$ These papers have concentrated on the properties of nanoalloys, whilst for most ionic nanoparticles computer simulations have largely investigated structure of binary nanoparticles, ${ }^{11}$ e.g. $\mathrm{TiO}_{2}$ clusters ${ }^{12}$ and nanotubes. ${ }^{13}$ Nevertheless addition of a third element is frequently used to control fundamental properties such as shape, growth and optical absorption. ${ }^{14}$ While Pandey and co-workers have worked systematically on compositional distributions in several ternary semiconductor quantum dots, ${ }^{15,16}$ relatively few papers ${ }^{17,18}$ have examined the distribution of ions and the associated thermodynamics of oxide nanoparticles despite their evident importance for a wide range of applications. ${ }^{19,20}$ Just one example is a key positive electrode material in Li-ion batteries, lithium iron phosphate $\left.(\mathrm{LiFePO})_{4}\right)$. Nanoscaling is essential for achieving high rate capability during electrochemical cycling and such olivine nanoparticles show very different phase transition behaviour from that of the bulk olivine phase..$^{21-26}$ Experimentally, the solubility of lithium in olivines ${ }^{21,24,27}$ ) increases with decreasing particle size.

Calculation of the thermodynamic properties of ternary nanoparticles is essential to develop our understanding of the distribution of elements within the nanoparticles and how this impacts on the properties of the nanoparticle. Indeed, the thermodynamic properties and chemical composition of a nanoparticle may determine its suitability for technological applications. 
For example, a miscibility gap may lead to the formation of a core-shell structure to the nanoparticle. In this paper we report results of Monte Carlo simulations to determine the structure and thermodynamic properties of nanoparticles comprised of a mixture of ionic materials, with initial application to small oxide nanoparticles of varying composition and where the cations differ appreciably in size.

For our pilot study $\mathrm{MgO}-\mathrm{MnO}$ is an appropriate choice of prototype system due to the magnitude of the difference in radius between the larger $\mathrm{Mn}^{2+}$ and the smaller $\mathrm{Mg}^{2+}$ ion (Shannon and Prewitt ionic radii in 6-fold coordination are $0.83 \AA$ and $0.72 \AA$, respectively). ${ }^{28}$ This mismatch is neither so small that an essentially ideal solution is formed at all compositions and temperatures nor so large that there is always complete segregation by one cation under all conditions of interest. $^{29}$ The bulk system has also received considerable attention previously. ${ }^{30}$ Our calculations explicitly include the effects of temperature and full surface relaxation, are not restricted to the dilute limit and there are no symmetry restraints or mean-field approximations. No assumptions are made as to the positions of the impurity/dopant atoms at the surfaces of the nanoparticles corresponding to a particular total composition; surface segregation and atomic relaxation in the ternary nanoparticle are automatically incorporated and as we shall play crucial roles.

\section{Methodology}

The surface of a crystal always provides a different elastic and electrostatic environment from that of the bulk, and so there is a free energy difference between the energy associated with any defect, including an impurity, in the bulk and at the surface of a nanoparticle. Impurities are thus driven to or from the surface. The basis for all our calculations is the well-known Monte Carlo (MC) method modified for nanoparticles of variable composition as described below. All nanoparticles were cubic and constructed from 8-atom units identical to the cubic unit cell of the bulk material. Calculations were undertaken on MnO-MgO mixtures comprising 1728 ions $(6 \times 6 \times 6)$, 2744 ions $(7 \times 7 \times 7), 4096$ ions $(8 \times 8 \times 8), 5832$ ions $(9 \times 9 \times 9), 8000$ ions $(10 \times 10 \times 10), 13824$ ions $(12 \times 12 \times 12)$ and 21952 ions $(14 \times 14 \times 14)$. We have considered only the $\{100\}$ termination of the nanoparticle since the $\{100\}$ surfaces of both $\mathrm{MgO}$ and $\mathrm{MnO}$ are considerably more stable than other terminations. $^{31}$ The rôle of both point defects (vacancies, interstitials) and linear defects (steps, dislocations) on the thermodynamics properties of the nanoparticles are neglected. We do not report results for smaller nanoparticles since the estimated consolute temperature is less than that at which exchange of ions in the simulations is very inefficient (approximately $500 \mathrm{~K}$ ).

All calculations were performed using the DL_MONTE program ${ }^{32}$ adapted for use with nanoparticles and were based on an ionic model using two-body potentials to represent short-range forces. ${ }^{33}$ The total long-range electrostatic energy was evaluated using direct summation (no periodic boundaries were employed). The particular non-Coulombic interaction potentials employed is the very widely used set of Lewis and Catlow first introduced in their study of the parent oxides, ${ }^{34}$ and implemented here with a cut-off of $12 \AA$. We have used these previously in studies of bulk $\mathrm{MnO}-\mathrm{MgO}$ solid solutions and the resulting phase diagram, calculated $^{35}$ using exchange Monte Carlo and the semigrand canonical ensemble is in good agreement with experiment with a consolute temperature of $\approx 1150 \mathrm{~K}$. In addition, we have successfully employed this model to calculate segregation energies $^{36}$ in thin films.

In simulations of non-stoichiometric ionic materials and solid solutions using 'standard' MC, kinetic barriers prevent sampling the whole of the configurational space since almost always only one cation arrangement - the initial configuration is sampled. We have described elsewhere ${ }^{37,38}$ Monte Carlo Exchange (MCX) simulations in which both the atomic configuration and the atomic coordinates of all the atoms are changed. A single cycle allows for one of three possible moves to be chosen at random with equal probability:

(i) random displacement of an ion. Allowing random moves of randomly selected atoms takes account of relaxation throughout the nanoparticle and vibrational effects. To determine whether the change is accepted or rejected, the usual Metropolis algorithm is applied. ${ }^{39}$ The maximum change in the atomic displacement for each ionic species has a variable $r_{\text {max }}$, its magnitude is adjusted automatically during the simulation to maintain an acceptance/rejection ratio of approximately 0.37 .

(ii) an exchange of ionic configuration. Changes in ionic configuration are attempted by exchanging the position of a $\mathrm{Mn}^{2+}$ ion and $\mathrm{a} \mathrm{Mg}^{2+}$ ion, both chosen at random. Again, the Metropolis algorithm is used to accept or reject any attempted exchange.

(iii) a change in identity of a cation (semigrand ensemble). ${ }^{40}$ We evaluate the potential energy change $\Delta U_{\mathrm{B} / \mathrm{A}}$ which would result if one species, $\mathrm{B}$ were to be converted into another, A. This change in energy is related to the corresponding change in chemical potential $\Delta \mu_{\mathrm{B} / \mathrm{A}}$ by,

$$
\Delta \mu_{\mathrm{B} / \mathrm{A}}=-k_{\mathrm{B}} T \ln \left\langle\frac{N_{\mathrm{B}}}{N_{\mathrm{A}}+1} \exp \left(-\Delta U_{\mathrm{B} / \mathrm{A}} / k_{\mathrm{B}} T\right)\right\rangle .
$$

Thus for MgO-MnO, we evaluate the energy associated with the conversion of a randomly chosen $\mathrm{Mg}$ ion to an $\mathrm{Mn}$ ion, $\Delta U_{\mathrm{Mg} / \mathrm{Mn}}$ and the reverse transformation. We emphasize that the change of $\mathrm{Mg}$ into $\mathrm{Mn}$, and vice versa, is only a temporary substitution to determine the free energy difference for a given $\mathrm{N}_{\mathrm{Mn}}$ and $\mathrm{N}_{\mathrm{Mg}}$; after $\Delta U_{\mathrm{Mg} / \mathrm{Mn}}$ is calculated, the Monte Carlo simulation of the nanoparticle continues with the initial composition. The semi-grand canonical ensemble is used in preference to the grand canonical since good statistics on $\Delta \mu_{\mathrm{B} / \mathrm{A}}$ can easily be achieved. To calculate the phase diagram, ${ }^{41}$ the calculated values of $\Delta \mu_{\mathrm{B} / \mathrm{A}}$ are fitted to eqn (2):

$$
\frac{\Delta \mu}{k_{\mathrm{B}} T}=\ln \left(\frac{x}{1-x}\right)+a x+b x^{2}+c x^{3} .
$$

By integrating eqn (2) with respect to composition we obtain the variation in free energy with $x$ at each temperature. Since we 
are only interested in free energies of mixing rather than absolute values the composition-independent constant of integration is irrelevant.

Each calculation consisted of an initial equilibration of $5 \times 10^{8}$ cycles followed subsequently by $5 \times 10^{8}$ production cycles. The larger clusters (>4096 ions) pose a significant computational challenge and for this reason the calculations were performed using a 8 to 128 cores in parallel replicated data strategy.

\section{Results and discussion}

We plot quantities of interest as a function of the total mole fraction of $\mathrm{Mg}^{2+}$. We first define, $\Delta H$, the enthalpy of forming ternary nanoparticles from the stoichiometric binary nanoparticles of the same size by the reaction,

$$
x \mathrm{Mg}_{n} \mathrm{O}_{n}+(1-x) \mathrm{Mn}_{n} \mathrm{O}_{n}=\mathrm{Mg}_{x n} \mathrm{Mn}_{(1-x) n} \mathrm{O}_{n},
$$

where $0 \leq x \leq 1$, and all the species in this equation are nanoparticles each containing $2 n$ ions (anions and cations in total).

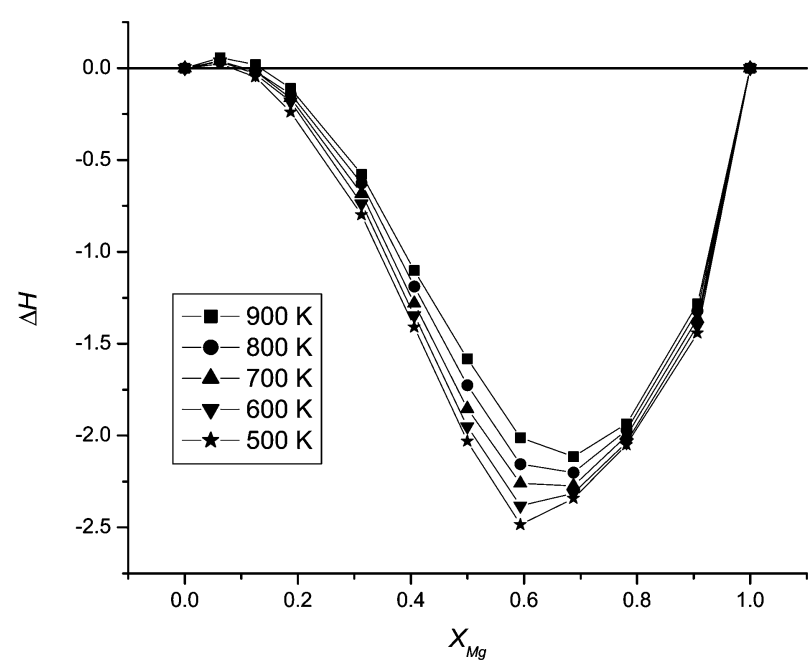

Fig. 1 Enthalpies of forming ternary nanoparticles, $\Delta H\left(\mathrm{~kJ} \mathrm{~mol}^{-1}\right)$, for the 1728 ion nanoparticle, defined in eqn (3), as a function of temperature and composition. $x_{\mathrm{Mg}}$ is the $\mathrm{Mg}$ mole fraction, as in eqn (3). The enthalpies in Fig. 1 and 3 are determined per cation to allow comparison with different sized nanoparticles.
$\Delta H$ is equal to the change in internal energy throughout this paper as the external pressure is zero. We consider below the variation of this quantity with composition and size of the nanoparticle. The enthalpies of forming ternary nanoparticles as a function of $\mathrm{Mg}$ concentration are displayed for the 1728 ion $(n=864)$ in Fig. 1 (this is determined as the energy per cation to allow comparison between different sized nanoparticles). We note that the enthalpy of forming ternary nanoparticles, as defined in eqn (3), is not positive over the entire composition range, but changes from being positive to negative at approximately $x_{\mathrm{Mg}}=0.125$. In contrast bulk MgO-MnO exhibits a positive enthalpy of mixing (defined for the bulk analogously to eqn (3)), over the entire composition range both experimentally and computationally. ${ }^{30,37}$

The variation of the enthalpy of forming ternary nanoparticles from nanoparticles of $\mathrm{MgO}$ and $\mathrm{MnO}$ comes from the relative energies of replacing a large cation $\left(\mathrm{Mn}^{2+}\right)$ with a small cation $\left(\mathrm{Mg}^{2+}\right)$ or the reverse. $\mathrm{Mg}-\mathrm{O}$ bonds are stronger (and stiffer) than Mn-O bonds and the $\{001\}$ surface energy of $\mathrm{MgO}$ larger than that of MnO. Thus when a few Mn ions are replaced by $\mathrm{Mg}$ in a $\mathrm{MnO}$ nanoparticle, the $\mathrm{Mg}$ ions are preferentially located within the internal structure of the nanoparticle. They are distributed randomly and do not form a cubewithin-cube structure (Fig. 2a-c). The $\mathrm{Mg}-\mathrm{O}$ bonds are stretched (static lattice simulations of a single $\mathrm{Mg}$ substitution indicate a $\mathrm{Mg}-\mathrm{O}$ bond length of approximately $2.17 \AA$ A; while that in the $\mathrm{MgO}$ nanoparticles is $2.10 \AA$ ). This strain gives rise to a positive enthalpy of forming ternary nanoparticles, and in the bulk material the positive enthalpy of mixing is positive for the same reason.

In contrast, if we start with a $\mathrm{MgO}$ nanoparticle and gradually replace the $\mathrm{Mg}$ ions by $\mathrm{Mn}$, the enthalpy of forming ternary nanoparticles is negative. This is due to the Mn ions progressively replacing $\mathrm{Mg}$ ions at the corners, edges and then in the surface of the nanoparticle (Fig. $2 \mathrm{~d}$ and e) and the driving force is the lower interface energy. We note in passing that replacement at corners is favoured rather than at edges in contrast to $\mathrm{Ca}^{2+}$ substitution in cubic $\mathrm{MgO}$ nanoclusters where the larger size mismatch and pronounced relaxation leads to the reverse behaviour. $^{42}$

For the 1728 ion nanoparticle, analysis of available sites at corners, edges and surfaces indicates that the nanoparticle is (a)

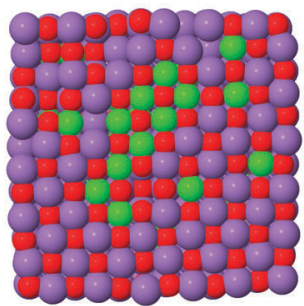

(b)

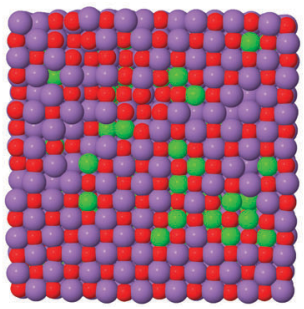

(c)

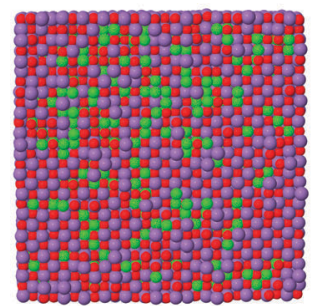

(d)

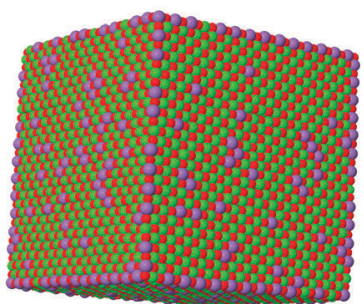

(e)

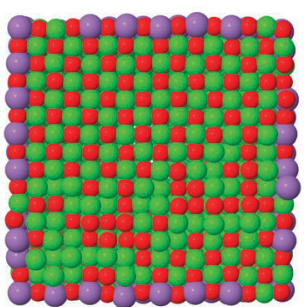

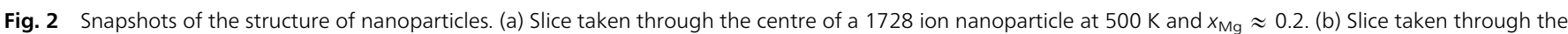

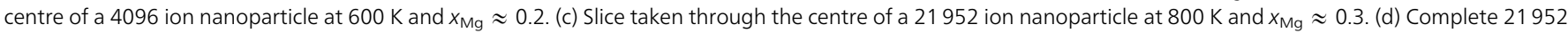

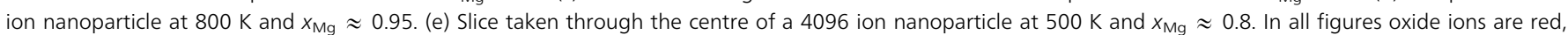
magnesium green and manganese purple. 
fully covered with $\mathrm{Mn}$ at approximately $x_{\mathrm{Mg}}=0.58$. This is very close to the minimum in the $500 \mathrm{~K} \Delta H v s$. composition curve in Fig. 1. The enthalpy of forming ternary nanoparticles is relatively insensitive to temperature, although the minimum decreases in magnitude (becomes less negative) and moves to slightly greater values of $x$ (to $x_{\mathrm{Mg}}=0.7$ at $900 \mathrm{~K}$ ). This trend results from the increase in temperature allowing greater numbers of Mn ions into subsurface layers.

While there is a pronounced preference of $\mathrm{Mn}^{2+}$ in the ternary nanoparticles for the outermost layers, there is no such marked preference for any other sites within the nanoparticles. Thus while there is $\mathrm{Mn}^{2+}$ enrichment at the surface, we do not observe the formation of nanoparticles containing an $\mathrm{MgO}$ "core" and an MnO outer "shell" consisting of more than just the surface layer (Fig. 2a-c).

In Fig. 3, we plot the enthalpy of forming ternary nanoparticles as a function of nanoparticle size. The region of positive $\Delta H$ becomes progressively greater in both magnitude and extent, while that where $\Delta H$ is negative becomes less negative and the position of the minimum moves to a larger value of $x_{\mathrm{Mg}}$. These changes arise due to the greater availability of 'bulklike' material as the size of the nanoparticle increases and the rôle of the surface becomes less important.

A phase diagram showing the stability of the ternary nanoparticles relative to separate binary nanoparticles of the same size (eqn (3)) can be determined using free energy differences from eqn (1) and (2). Fig. 4a-c display the calculated values of $\Delta \mu_{\mathrm{Mg} / \mathrm{Mn}}$ for the 1728, 8000 and 21952 ion structures respectively and represent typical behaviour (intermediate size nanoparticles display similar behaviour between the extremes). The existence of a minimum and maximum in a $\Delta \mu_{(x)}$ curve indicates a miscibility gap at that temperature. For the 1728 nanoparticle it is clear from the shape of these curves that $500 \mathrm{~K}$ and $600 \mathrm{~K}$ corresponds to temperatures below the consolute temperature $T_{\mathrm{C}}$ (stationary points are evident), and the formation of one-ternary-particle and two-binary-particle

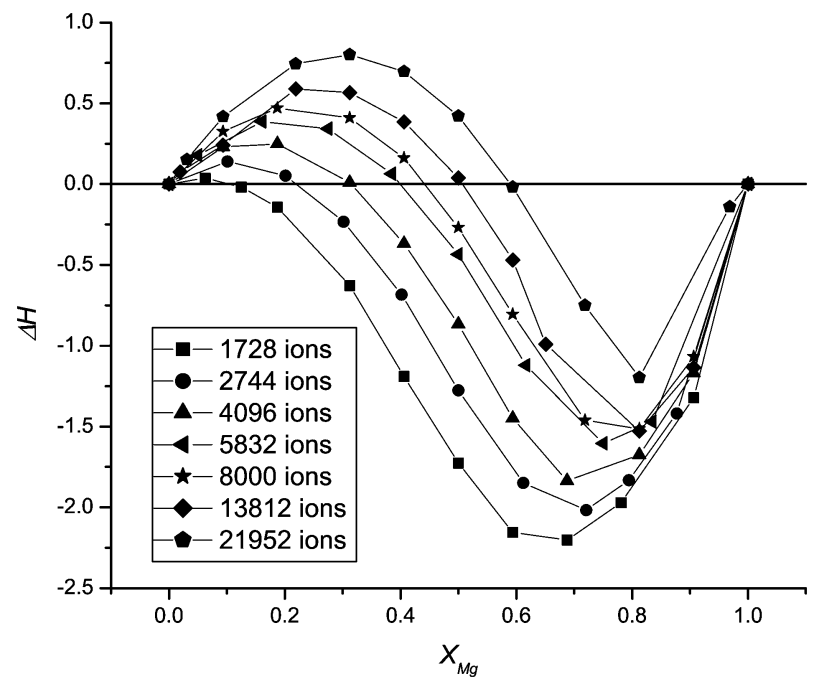

Fig. 3 Calculated values of $\Delta H$ (eqn (3)) $\left(\mathrm{kJ} \mathrm{mol}^{-1}\right)$ for different size nanoparticles at $800 \mathrm{~K}$.
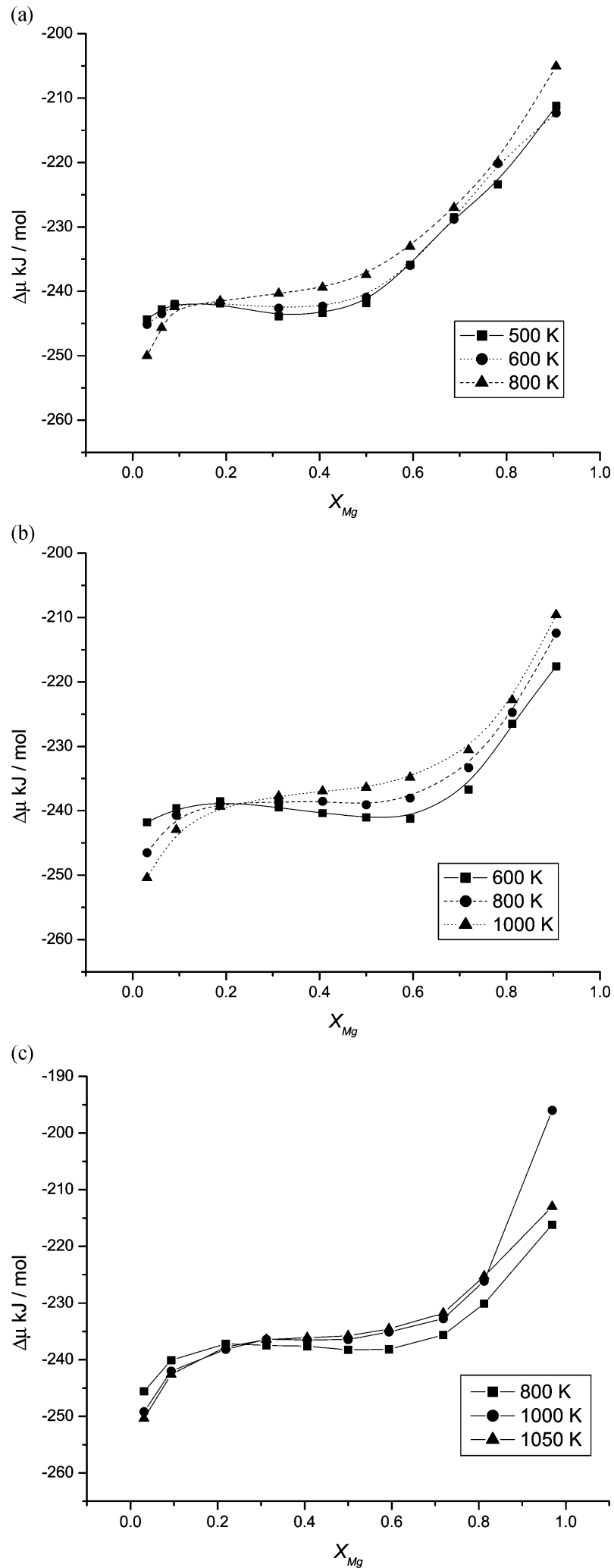

Fig. 4 Calculated values of $\Delta \mu_{\mathrm{Mg} / \mathrm{Mn}}$ (eqn (2)) for the (a) 1728 , (b) 8000 and (c) 21952 ion structures.

regions at different compositions. The $\Delta \mu_{(x)}$ curve for $600 \mathrm{~K}$, is just below the calculated $T_{\mathrm{C}}$, as indicated by the shape of the 
curve in which the stationary points evident at $500 \mathrm{~K}$ have almost disappeared. At $800 \mathrm{~K}$ the stationary points are not present and this temperature is above the consolute temperature. In contrast, calculated values of $\Delta \mu_{\mathrm{Mg} / \mathrm{Mn}}$ for the 8000 and
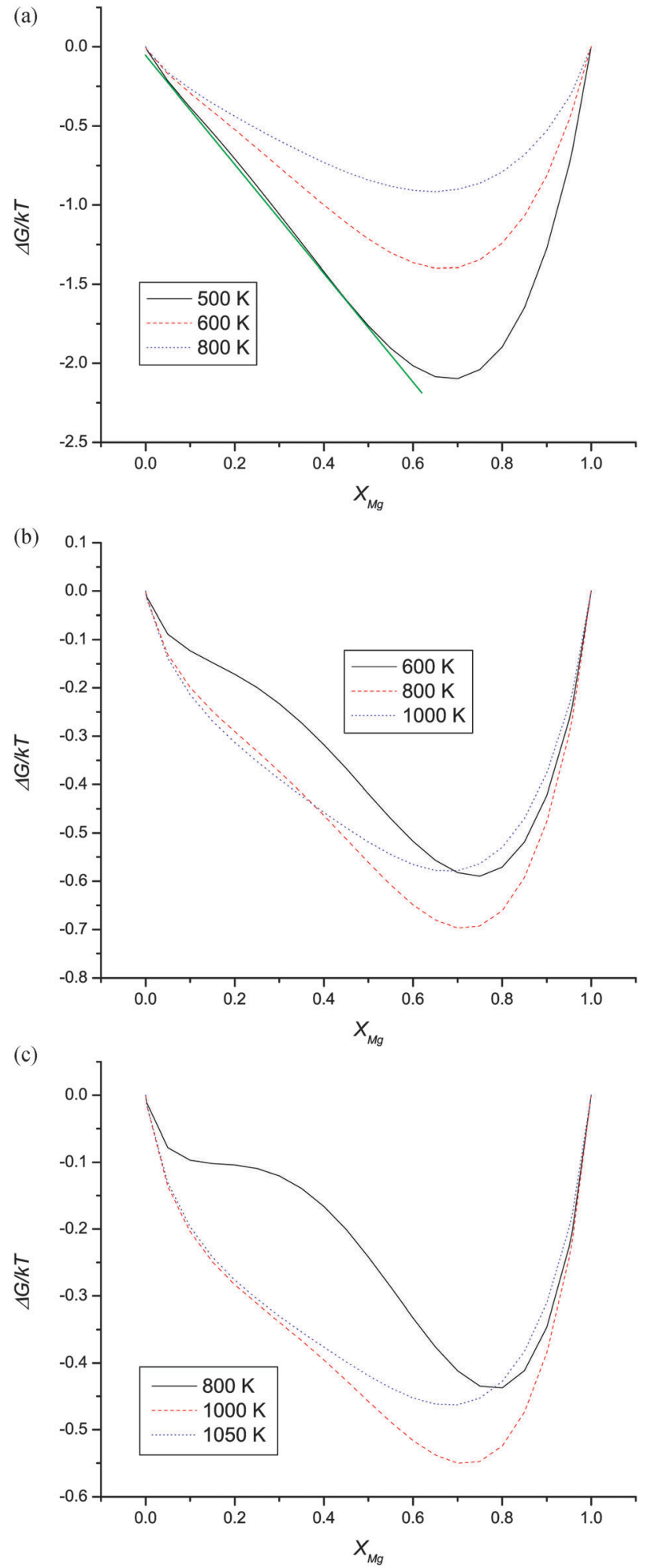

Fig. $5 \Delta G$ (eqn (3)) for the (a) 1728, (b) 8000 and (c) 21952 ion structures In Fig. $5 a$ the straight line is a guide to the eye.
21952 ion nanoparticles still exhibit weak stationary points at $800 \mathrm{~K}$ and $1000 \mathrm{~K}$ respectively and we would still expect the

(a)

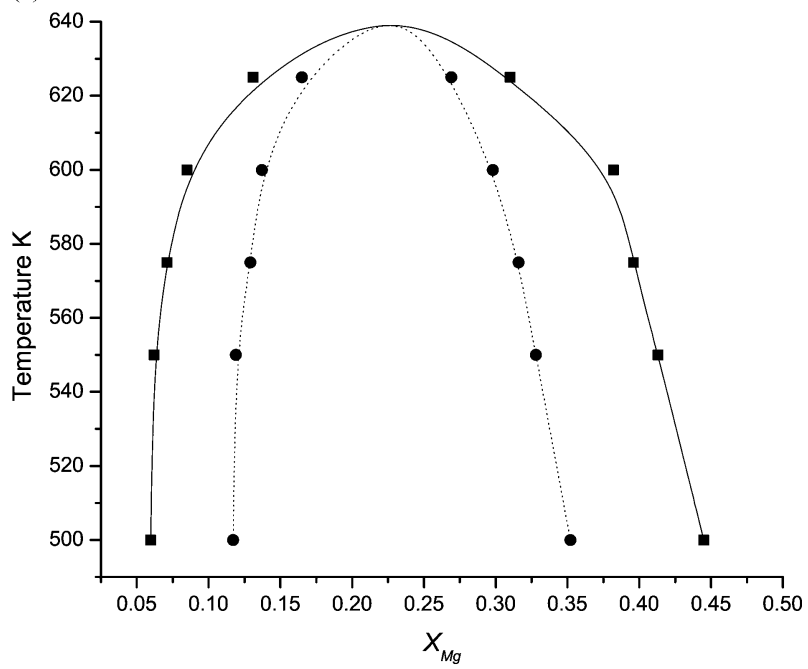

(b)

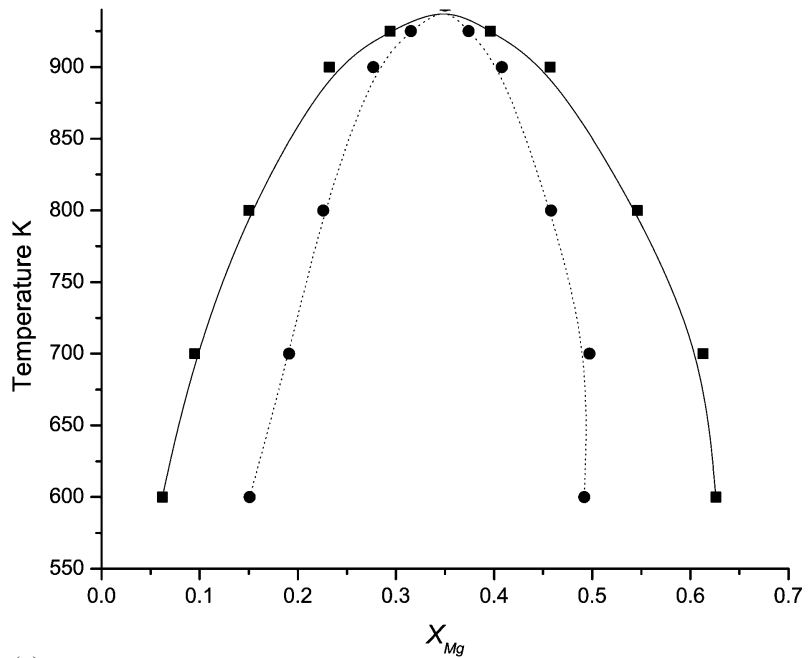

(c)

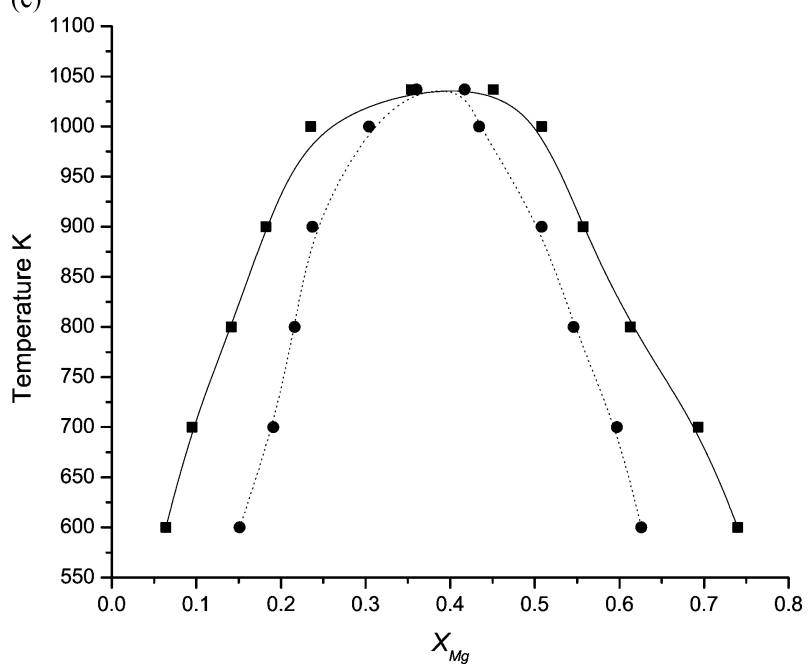

Fig. 6 Phase diagrams for the (a) 1728, (b) 8000 and (c) 21952 ion nanoparticles. Solid squares $=$ binodal, solid diamond $=$ spinodal. 
formation of one-ternary particle and two-binary particle regions at different compositions. For these sizes of nanoparticle, the stationary points are not present at $1000 \mathrm{~K}$ and $1050 \mathrm{~K}$ and the temperature is above $T_{\mathrm{C}}$.

In Fig. $5 \mathrm{a}$ and $\mathrm{b}$ we plot calculated values of $\Delta G$ for the forming ternary nanoparticles (as defined in eqn (3)) vs. $x$ obtained by integration of eqn (2) at temperatures 500, 600 and $800 \mathrm{~K}$ and 600,800 and $1000 \mathrm{~K}$ for the 1728 and 8000 ion nanoparticles respectively. Minima in $\Delta G$ at any particular temperature indicate that one-ternary-particle and two-binaryparticle regions are formed at different compositions and hence such temperatures lies below $T_{\mathrm{C}}$. For the smaller nanoparticle (Fig. 5a), the two minima in $\Delta G$ observed at $500 \mathrm{~K}$ and $600 \mathrm{~K}$ curve are very weak (especially for $600 \mathrm{~K}$ as it is just below $T_{\mathrm{C}}$ ) and hardly detectable by eye in the figure. At temperatures greater than $640 \mathrm{~K}$, as indicated by the $800 \mathrm{~K}$ curve, the dependence of $\Delta G$ at all compositions is consistent with complete miscibility. For the larger nanoparticles the minima in the curves are more evident and indicate the oneternary and two-binary regions of the phase diagram are still present at higher temperatures (e.g., the 600 and $800 \mathrm{~K}$ curves for the 8000 ion nanoparticle and 800 and $1000 \mathrm{~K}$ curves for the 21952 ion nanoparticle). The curves at 1000 and $1050 \mathrm{~K}$ are, however, consistent with complete miscibility for the 8000 and 21952 ion nanoparticles respectively. Given curves as in Fig. 5, a common tangent construction at each temperature yield the phase diagrams in Fig. $6 a$ and b. The behaviour of the intermediate size nanoparticles exhibit a gradation in their behaviour. For comparison the calculated and experimental phase diagram of bulk $\mathrm{MgO}-\mathrm{MnO}$ is presented in Fig. 7. Thus by performing calculations at several temperatures for the different size nanoparticles, $T_{\mathrm{C}}$ can be determined as a function of the number of ions in the nanoparticle (Fig. 8). The variation with the total number of ions is nonlinear. However, limitations in the range of nanoparticle size and sampling of the Monte Carlo method have prevented us from determining whether the curve is sigmoidal as

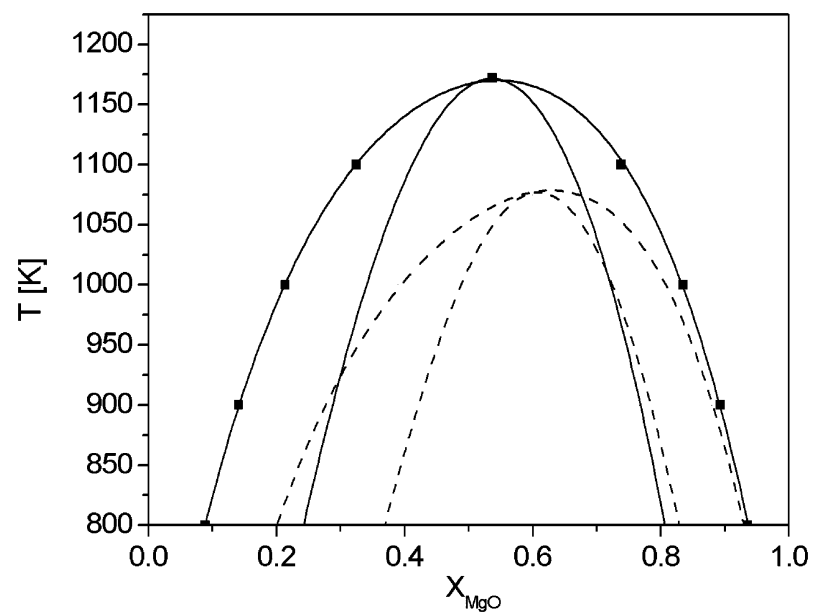

Fig. 7 Calculated (solid lines) and experimental (dashed lines) phase diagrams, and spinodals for bulk MnO-MgO. The experimental results are from ref. 45 .

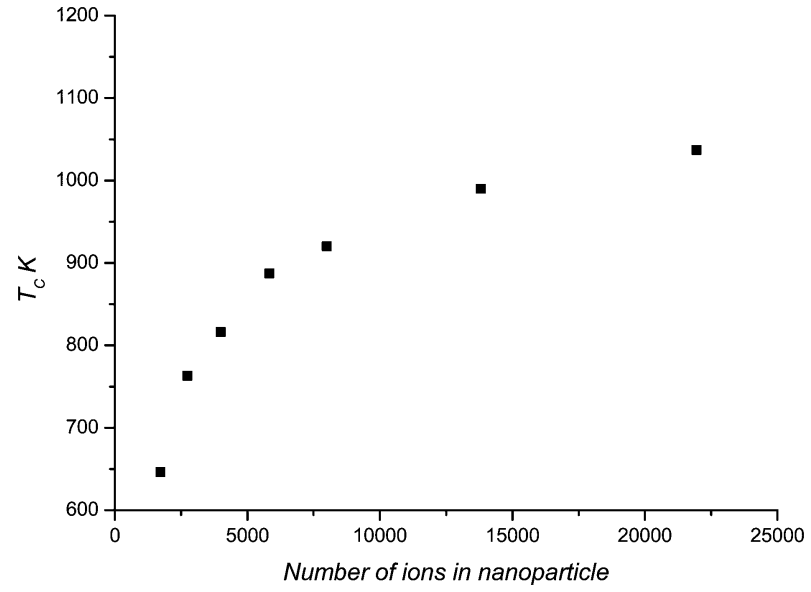

Fig. 8 The consolute temperature $T_{C}$ as a function of particle size (total number of ions).

observed in previous studies. ${ }^{30}$ All the phase diagrams are slightly asymmetric.

The calculated phase diagrams for nanoparticles of the same size (eqn (3)) show that at low temperatures $(<600 \mathrm{~K})$ the $\mathrm{MgO}$ solubility in $\mathrm{MnO}$ is low $\left(x_{\mathrm{Mg}}=0.05-0.07\right)$ and does not vary much between the different nanoparticles and the bulk. In contrast at the same temperatures the solubility of $\mathrm{MnO}$ in $\mathrm{MgO}$ is much higher in the smaller nanoparticles (as high as $x_{\mathrm{Mn}}=0.4$ at $600 \mathrm{~K}$ even for the 8000 -ion particle (driven by the interface energies); there is a rapid decrease as the size of the nanoparticle increases. The interface term here enhances the common trend that the compound containing the smaller ion is usually more soluble in the compound with the larger ion than the reverse.

\section{Conclusions}

We have employed the Monte Carlo Exchange technique to calculate directly the thermodynamic properties of $\mathrm{MnO}-\mathrm{MgO}$ clusters as a function of temperature and nanoparticle size (between 1728 and 21952 ions). As the Mn concentration is progressively increased the $\mathrm{Mn}^{2+}$ ions occupy the corners, edges and then surface sites of the nanoparticle. The enthalpy of forming ternary nanoparticles (eqn (3)) depends on the size of the nanoparticle and for given concentration becomes more positive as the particle size increases. Where the enthalpy is negative, the different interface energies of $\mathrm{MnO}$ and $\mathrm{MgO}$ provide the driving force. Application of the semigrand ensemble has allowed us to determine the mixing free energies of the two end-member nanoparticles. In addition, we find that the consolute temperature initially increases rapidly as a function of nanoparticle size. The rate of increase is reduced for larger nanoparticles. Similar trends were observed for the order parameter in FePt nanoparticles ${ }^{7,43}$ and the melting properties of PtPd nanoalloys. ${ }^{8}$ It is important to note that the ternary nanoparticles themselves may well be highly ordered with a shell of $\mathrm{MnO}$ around a central $\mathrm{MgO}$ core, particularly at $x_{\mathrm{Mg}} \approx$ 0.8-0.6 concentrations and low temperatures; the different 
interface energies of $\mathrm{MgO}$ and $\mathrm{MnO}$ provide the thermodynamic barrier to forming separate binary nanoparticles. It will be interesting to see to what extent the trends we establish in this paper are more general when size mismatch governs thermodynamic properties of mixing. Our Monte Carlo method is general and we shall apply this in future work to interfaces of technological importance. In particular, we intend to study more complex nanoparticles and explore the influence of changes in surface environment such as hydroxylation.

\section{Acknowledgements}

Computing resources were provided by STFC's e-science facility. Fig. 2 was prepared using Jmol. ${ }^{44}$

\section{References}

1 B. Kang and G. Ceder, Nature, 2009, 458, 190.

2 B. C. Gates, L. Guczi and H. Knoezinger, Metal Clusters in Catalysis, Elsevier, Amsterdam, 1986.

3 R. Ferrando, J. Jellinek and R. L. Johnston, Chem. Rev., 2008, 108, 845 .

4 For example, R. Vallée, M. Wautlet, J. P. Dauchot and M. Hecq, Nanotechnology, 2001, 12, 68.

5 T. Van Hoof and M. Hou, Phys. Rev. B: Condens. Matter Mater. Phys., 2005, 72, 115434.

6 D. S. Mainardi and P. B. Balbuena, Langmuir, 2001, 17, 2047.

7 M. Müller and K. Albe, Phys. Rev. B: Condens. Matter Mater. Phys., 2005, 72, 094203.

8 G. Wang, M. A. Van Hove, P. N. Ross and M. I. Baskes, J. Chem. Phys., 2004, 121, 5410.

9 G. Guisbiers, G. Abudukelimu and D. Hourlier, Nanoscale Res. Lett., 2011, 6, 396.

10 H. Y. Kim, D. H. Kim and H. M. Lee, J. Nanosci. Nanotechnol., 2011, 11, 2251.

11 For a recent review see C. R. A. Catlow, S. T. Bromley, S. Hamad, M. Mora-Fonz, A. A. Sokol and S. M. Woodley, Phys. Chem. Chem. Phys., 2010, 12, 786.

12 For example, S. M. Woodley, Proc. R. Soc. A, 2011, 467, 2020.

13 For example, J. N. Hart, S. C. Parker and A. A. Lapkin, ACS Nano, 2009, 3, 3401.

14 D. G. Stroppa, L. A. Montoro, A. Beltran, T. G. Conti, R. O. da Silva, J. Andres, E. R. Leite and A. J. Ramirez, Chem.-Eur. J., 2011, 17, 11515.

15 For example, S. C. Pandey and D. Maroudas, J. Chem. Phys., 2011, 135, 23470 and references therein.

16 S. C. Pandey and D. Maroudas, J. Appl. Phys., 2011, 110, 123509 and references therein.

17 S. M. Woodley, Mater. Manuf. Processes, 2009, 24, 255.

18 X. Wang, J. C. Hanson, J. A. Rodriguez, C. Belver and M. Fernández-García, J. Chem. Phys., 2005, 122, 154711.

19 For work on phase segregation in $\mathrm{SiO}_{2} / \mathrm{TiO}_{2}$ and $\mathrm{SiO}_{2} / \mathrm{Fe}_{2} \mathrm{O}_{3}$ nanoparticle aerosols, see S. H. Ehrman, S. K. Friedlander and M. R. Zachariah, J. Mater. Res., 1999, 14, 4553.
20 For work on $\mathrm{SnO}_{2} / \mathrm{TiO}_{2}$ composite nanoparticles, K. K. Akurati, A. Vital, R. Hany, B. Bommer, T. Graule and M. Winterer, Int. J. Photoenergy, 2005, 2, 153.

21 N. Meethong, H. Y. S. Huang, W. C. Carter and Y.-M. Chiang, Electrochem. Solid-State Lett., 2007, 10, A134.

22 N. Meethong, H. Y. Huang, S. A. Speakman, W. C. Carter and Y.-M. Chiang, Adv. Funct. Mater., 2007, 17, 1115.

23 A. Yamada, H. Koizumi, S.-I. Nishimura, N. Sonoyama and R. Kanno, et al., Nat. Mater., 2006, 5, 357.

24 P. Gibot, M. Casas-Cabanas, L. Laffont, S. Levasseur and P. Carlach, et al., Nat. Mater., 2008, 7, 741.

25 N. Meethong, Y.-H. Kao, W. C. Carter and Y.-M. Chiang, Chem. Mater., 2010, 22, 1088.

26 M. Tanga, W. C. Carter, J. F. Belak and Y.-M. Chiang, Electrochim. Acta, 2010, 56, 969.

27 B. Burch and M. Z. Bazant, Nano Lett., 2009, 9, 3795.

28 R. D. Shannon, Acta Crystallogr., Sect. A: Cryst. Phys., Diffr., Theor. Gen. Crystallogr., 1976, 32, 751.

29 J. A. Purton and N. L. Allan, J. Cryst. Growth, 2006, 294, 130.

30 N. L. Allan, G. D. Barrera, M. Yu. Lavrentiev, I. T. Todorov and J. A. Purton, J. Mater. Chem., 2001, 11, 63.

31 W. C. Mackrodt, J. Chem. Soc., Faraday Trans., 1989, 2, 541. 32 http://www.ccp5.dl.ac.uk/DLMONTE.

33 C. R. A. Catlow and W. C. Mackrodt, in Computer Simulation of Solids, ed. C. R. A Catlow and W. C. Mackrodt, SpringerVerlag, Berlin, 1982, ch. 1, pp. 3-20.

34 G. V. Lewis and C. R. A. Catlow, J. Phys. C: Solid State Phys., 1985, 18, 1149.

35 N. L. Allan, G. D. Barrera, R. M. Fracchia, M. Yu. Lavrentiev, M. B. Taylor, I. T. Todorov and J. A. Purton, Phys. Rev. B: Condens. Matter Mater. Phys., 2001, 63, 094203; I. T. Todorov, N. L. Allan, M. Yu. Lavrentiev, C. L. Freeman, C. E. Mohn and J. A. Purton, J. Phys.: Condens. Matter, 2004, 16, S2751.

36 J. A. Purton, M. Y. Lavrentiev and N. L. Allan, Phys. Chem. Chem. Phys., 2005, 7, 3601.

37 J. A. Purton, G. D. Barrera, M. B. Taylor, N. L. Allan and J. D. Blundy, J. Phys. Chem. B, 1998, 102, 5202.

38 For an example application to ordering in spinels, see M. Yu. Lavrentiev, J. A. Purton and N. L. Allan, Am. Mineral., 2003, 88, 1522.

39 N. I. Metropolis, A. W. Rosenbluth, M. N. Rosenbluth, A. H. Teller and E. Teller, J. Chem. Phys., 1953, 21, 1087.

40 D. Frenkel and B. Smit, Understanding Molecular Simulation, 2nd edn, Academic Press, San Diego-London, 2002.

41 M. Y. Lavrentiev, N. L. Allan, G. D. Barrera and J. A. Purton, J. Phys. Chem. B, 2001, 105, 3594.

42 C. E. Mohn, N. L. Allan and J. H. Harding, Phys. Chem. Chem. Phys., 2009, 11, 3217; K. McKenna, private communication.

43 M. Müller, P. Erhart and K. Albe, Phys. Rev. B: Condens. Matter Mater. Phys., 2007, 76, 155412.

44 Jmol: an open-source Java viewer for chemical structures in 3D, http://www.jmol.org/.

45 B. J. Wood, R. T. Hackler and D. P. Dobson, Contrib. Mineral. Petrol., 1994, 115, 438. 